\title{
New intervention in mitral valve prolapse Use of cold pressor test during echocardiography
}

\author{
DAVID PITCHER*, MICHAEL TYNAN, RAY WAINWRIGHT, PAUL CURRY, \\ EDGAR SOWTON
}

From the Department of Cardiology, Guy's Hospital, London

SUMMARY A study was performed in 13 patients with idiopathic mitral valve prolapse and in 21 control subjects to assess the effect of the cold pressor test on systolic motion of the mitral valve. A significant increase in blood pressure occurred in 10 patients with mitral valve prolapse and in 19 controls after immersion of one hand in ice-cold water. M-mode echocardiographic recordings from the mitral valve were obtained in all patients before, during, and after the cold pressor test, together with simultaneous phonocardiograms in selected patients. Nine out of 10 patients with mitral valve prolapse and a hypertensive response to the cold stimulus showed a significant increase in the depth of mitral valve prolapse during the cold pressor test whereas in three patients with mitral valve prolapse and no hypertensive response the depth of mitral valve prolapse did not change during cold stimulation. Three patients with previously demonstrated mitral valve prolapse had equivocal resting echocardiograms but developed diagnostic evidence of mitral valve prolapse during the cold pressor test. No evidence of mitral valve prolapse was seen in any of the control subjects before, during, or after the cold pressor test.

In four patients with mitral valve prolapse and a hypertensive response to cold stimulation the systolic click was delayed by the cold pressor test, whereas the time of the systolic click remained constant in the three patients whose blood pressure did not increase.

It is concluded that the cold pressor test provides a stimulus sufficient to delay the onset and increase the depth of mitral valve prolapse, thereby enhancing the diagnostic sensitivity of echocardiography in this condition.

Echocardiography is the most important technique for the non-invasive diagnosis of mitral valve prolapse. Despite careful echocardiographic examination at rest, however, some patients with mitral valve prolapse have normal or non-diagnostic mitral echograms. In some patients this may be a result of localised prolapse occurring in a part of the mitral valve inaccessible to the ultrasound beam. In other patients shallow prolapse of the mitral valve at the time of the examination may fail to give rise to diagnostic echocardiographic abnormalities. In the latter situation particularly, certain physiological and pharmacological interventions may increase the diagnostic sensitivity of echocardiography. Such manoeuvres as head-up tilt, Valsalva, and amyl nitrite inhalation exaggerate the echocardiographic features of mitral valve prolapse and alter the timing

^Present address: Plymouth General Hospital, Plymouth, South Devon.

Received for publication 26 March 1980 of its onset, ${ }^{12}$ but these manoeuvres may produce the echocardiographic appearances of mitral valve prolapse in normal people. ${ }^{3}$

The cold pressor test ${ }^{4}$ has not previously been described as a diagnostic intervention in patients with mitral valve prolapse. In the present study we examined the effect of this stimulus on the echocardiographic and phonocardiographic findings in patients with mitral valve prolapse and in a group of control subjects.

\section{Patients and methods}

Thirteen patients with idiopathic mitral valve prolapse and 21 control subjects were studied. Details of the patients with mitral valve prolapse are shown in the Table. All had clinical signs suggesting mitral valve prolapse which had been confirmed previously by echocardiography in all cases. Four patients had selective coronary arterio- 
Table Clinical details of patients with mitral valve prolapse

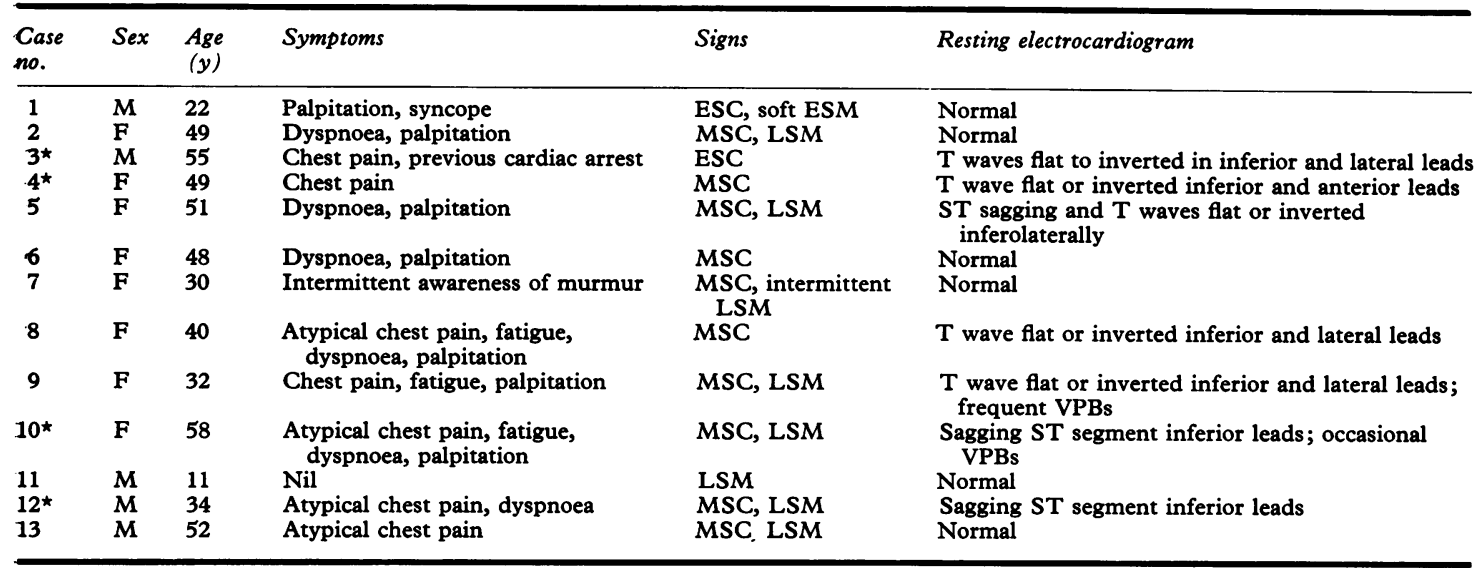

ESC, early systolic click; ESM, ejection systolic murmur; MSC, mid-systolic click; LSM, late-systolic murmur; VPB, ventricular premature beat. * Coronary arteriography performed (normal).

graphy which was normal and contrast left ventriculography which further confirmed mitral valve prolapse. Cardiac catheterisation was not performed in the remainder, in whom coronary artery disease was not suspected clinically. The control group comprised six patients with angiographically proven coronary artery disease, two patients with non-obstructive hypertrophic cardiomyopathy, and 13 normal subjects.

All patients were studied while resting on a couch

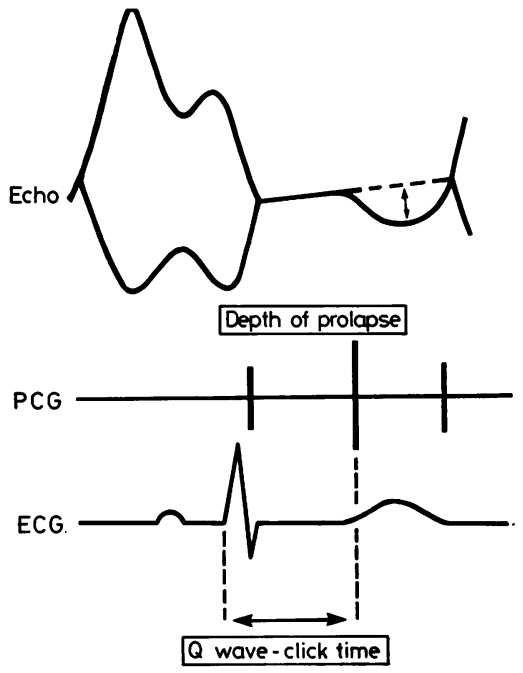

Fig. 1 The measured depth of mitral valve prolapse (small arrow) is shown on a diagrammatic echocardiogram, with phonocardiogram (PCG) and electrocardiogram (ECG) illustrating the $Q$ wave-systolic click time (large arrow). in the $30^{\circ}$ left oblique position. Using a Dinamap automatic sphygmomanometer, readings of heart rate and blood pressure were obtained at one-minute intervals throughout the study, and were recorded by an independent observer. M-mode echocardiograms were recorded from the mitral valve at the same intervals, using an Ekoline 20A ultrasonoscope with a $2.25 \mathrm{MHz}$ transducer focused at $10 \mathrm{~cm}$ and maintained in a constant position by hand. The transducer was initially aligned so as to show the maximum possible depth of mitral valve prolapse in each patient. Simultaneous phonocardiograms were recorded in seven patients. All recordings were made during a three-minute pretest period, during five minutes with the patient's hand immersed in ice-cold water, and for three minutes afterwards.

The echocardiograms were analysed without knowledge of the timing of a given recording or of the blood pressure response in each patient. The depth of mitral valve prolapse was measured from the echocardiograms as the maximum depth of posterior displacement of the mitral echo during systole (Fig. 1). On each recording the depth of mitral valve prolapse was taken as the average of measurements made over five consecutive cardiac cycles, thus minimising the effect of respiratory movement. Where phonocardiograms were obtained the time from the initial QRS deflection on the electrocardiogram to the systolic click on the phonocardiogram ( $Q$ wave-click time) was also measured and averaged over five consecutive cycles.

Analysis of variance was used to assess the statistical significance of the results. The null hypothesis was rejected when its probability was less than 0.05 . 


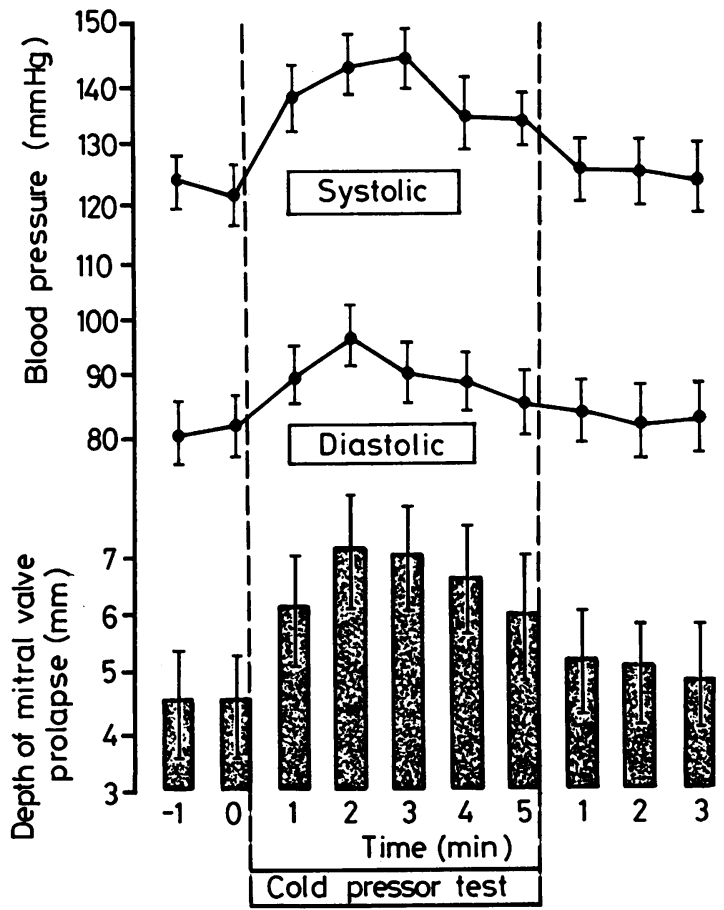

Fig. 2 Mean systolic and diastolic blood pressures (SE) and mean depth of mitral valve prolapse in 10 patients with mitral valve prolapse who showed a pressor response to cold.

\section{Results}

Ten out of 13 patients with mitral valve prolapse showed a hypertensive response to the cold stimulus (Fig. 2) and during cold stimulation nine of these developed a significant increase in the depth of prolapse $(p<0.001)$, which had returned to resting or near-resting levels three minutes after removal of the cold stimulus (Fig. 2 and 3). Furthermore, in three patients with previously documented mitral valve prolapse the resting echocardiograms on the day of the study were equivocal. During the cold pressor test abrupt posterior motion of the mitral echo in mid-systole, diagnostic of mitral valve prolapse, was seen in these three patients. In contrast, three patients with mitral valve prolapse had no hypertensive response to cold, and no significant change in the depth of mitral valve prolapse occurred during the study. In the control group 19 had a hypertensive response but none showed evidence of mitral valve prolapse before, during, or after the cold pressor test. In two control subjects no hypertensive response was observed and the mitral echograms remained normal.
Phonocardiograms were obtained from four patients with mitral valve prolapse who experienced a pressor response to cold stimulation. In all four there was a transient increase in $\mathrm{Q}$ wave-click time of 30 to $50 \mathrm{~ms}$ (mean $35 \mathrm{~ms}$ ) during the cold pressor test (Fig. 4) and the intensity of the late systolic murmur increased in one patient. In the three patients with mitral valve prolapse who had phonocardiograms recorded but who showed no hypertensive response the $Q$ wave-click time remained constant.

In 11 patients with mitral valve prolapse the heart rate increased during cold stimulation. Ten had increases of one to eight beats per minute and one patient had an increase of 18 beats per minute. Increases in heart rate tended to precede the in-

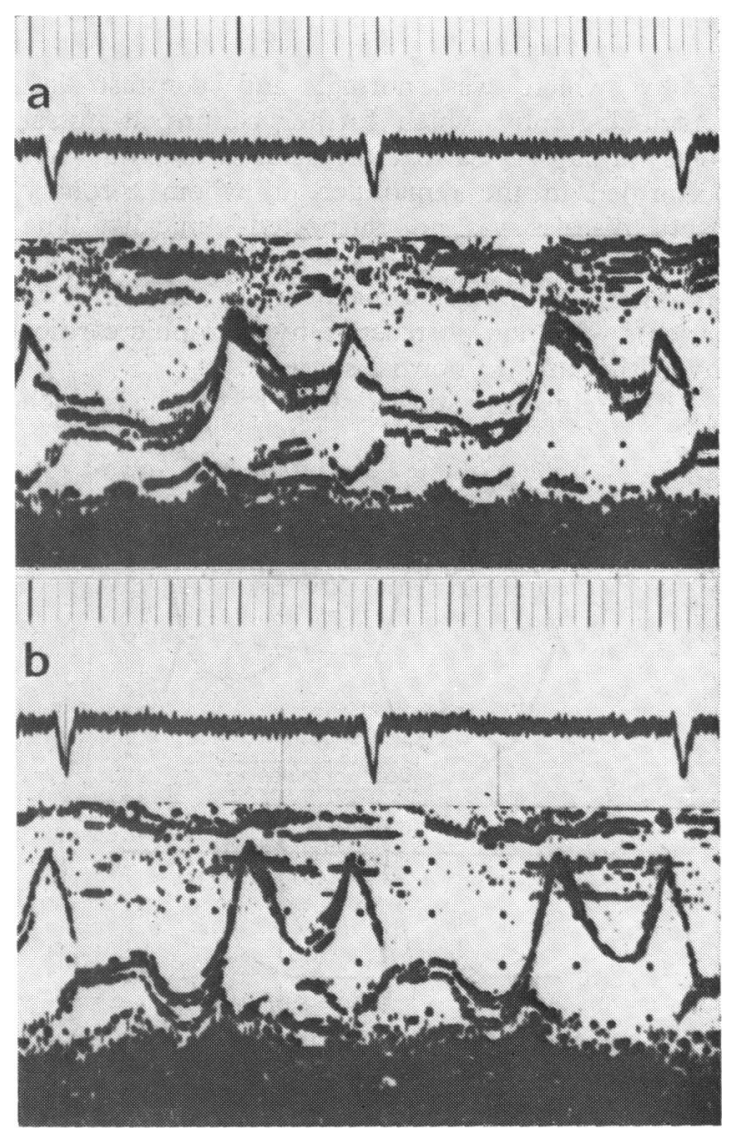

Fig. 3 Mitral echograms from a patient with mitral valve prolapse: (a) before the cold pressor test showing pansystolic posterior sagging of the dominant echoes from the mitral valve and (b) during the cold pressor test showing severe mid-systolic buckling of the mitral echo. 


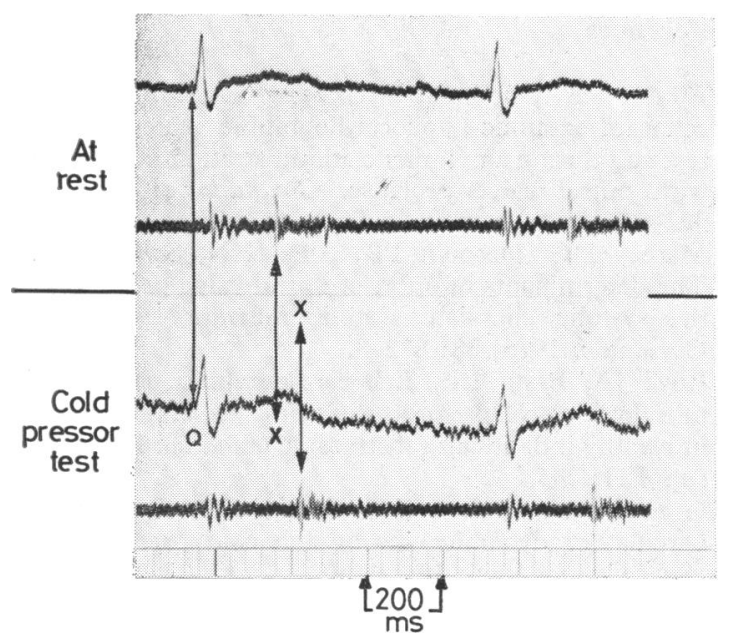

Fig. 4 Simultaneous electrocardiograms and phonocardiograms from a patient with mitral valve prolapse. In the upper trace, recorded at rest, the $Q R S$ complex has been aligned with that of the lower trace, recorded during cold pressor test. The delay in the systolic click caused by the cold pressor test is indicated by the two arrows (X).

creases in blood pressure: the magnitude of the heart rate increase showed no relation to the magnitude of the pressor response or the increase in depth of prolapse. Two patients showed a pressor response with increased depth of mitral valve prolapse in the presence of a slight fall in heart rate during cold stimulation.

\section{Discussion}

Patients with mitral valve prolapse may be asymptomatic but many present with symptoms such as chest pain, dyspnoea, palpitation, or syncope, usually out of proportion to any measurable haemodynamic abnormality. The characteristic clinical signs of a systolic click, with or without a late systolic murmur, may suggest the diagnosis. Some patients, however, have no abnormal physical signs. ${ }^{5}$ In this situation echocardiography is the only non-invasive means of diagnosing mitral valve prolapse, but even the echocardiographic signs may be intermittent or equivocal. While abrupt posterior motion of the mitral valve echo in midsystole is specific for mitral valve prolapse the distinction between holosystolic mitral valve prolapse and non-specific systolic sagging of the mitral echo is less obvious. Physiological and pharmacological manoeuvres may be used to alter the timing of onset of the mitral valve prolapse seen on the echocardiogram and of the closely related systolic click. ${ }^{12}$ Mathey et al. ${ }^{2}$ have shown that the onset of mitral valve prolapse occurs at a relatively fixed ventricular dimension in individual patients. Thus, a delay in onset of the mitral valve prolapse, as seen in our patients during the cold pressor test, may be explained either by an increase in left ventricular end-diastolic dimension or by a reduction in left ventricular performance before the onset of prolapse. In either situation the left ventricle would take longer to reach the critical dimension at which prolapse occurs. Our results do not, however, distinguish between these two mechanisms, as left ventricular dimensions were not measured in this study focusing as it did primarily on mitral valve motion.

In our patients the depth of mitral valve prolapse was increased during the cold pressor test. Devereux et al. ${ }^{6}$ suggest that manoeuvres that reduce left ventricular cavity size cause mitral valve prolapse to be both earlier and greater and that those that increase left ventricular cavity size result in later and lesser prolapse. But mitral valve prolapse was both later and greater during the cold pressor test, an intervention that increases blood pressure and that would not be expected to reduce left ventricular volume in the absence of consistent increases in heart rate. This suggests that the depth of mitral valve prolapse is not entirely dependent on left ventricular dimensions, and our results showed a relation between increases in blood pressure and depth of mitral valve prolapse. Further evidence that the depth of mitral valve prolapse is not determined solely by left ventricular dimensions may be obtained by examining the motion of the mitral valve echo in mitral valve prolapse. The depth of prolapse begins to decrease immediately after the aortic component of the second heart sound (aortic valve closure), as left ventricular pressure falls during isovolumic relaxation (D W Pitcher, unpublished observations). It therefore seems probable that while the onset of mitral valve prolapse is determined by left ventricular dimensions, the depth of prolapse is determined, at least in part, by left ventricular systolic pressure.

Like other manoeuvres causing deeper mitral valve prolapse, the cold pressor test increases the sensitivity of echocardiographic detection of prolapse. However, Valsalva, head-up tilting, or amyl nitrite inhalation cause earlier as well as deeper prolapse. These manoeuvres may convert the specific echo pattern of mid-systolic prolapse to the less specific holosystolic prolapse, and may also produce echocardiographic appearances of mitral valve prolapse in normal people. ${ }^{3}$ No such abnormalities were caused by the cold pressor test 
in our control group. By causing later as well as deeper prolapse, the cold pressor test will tend to convert a non-specific echogram showing holosystolic sagging of the mitral echo to the specific pattern of mid-systolic mitral valve prolapse and may thereby enhance the sensitivity of echocardiography in mitral valve prolapse without loss of specificity.

As a result of these studies we feel that the cold pressor test is indicated during echocardiography in patients presenting with unexplained chest pain, arrhythmias, or isolated late-systolic murmurs, in whom the echocardiogram is normal or equivocal at rest. Detection of otherwise "silent" mitral valve prolapse in this way may prevent needless further investigation. Further studies in such patients are indicated to assess the sensitivity of the cold pressor test in provoking echocardiographic evidence of mitral valve prolapse when the resting echocardiogram fails to detect it.

We thank Messrs P K Morgan Ltd. for the use of the automatic sphygmomanometer in this study.

\section{References}

1 Winkle RA, Goodman DJ, Popp RL. Simultaneous echocardiographic-phonocardiographic recordings at rest and during amyl nitrite administration in patients with mitral valve prolapse. Circulation 1975; 51: $522-9$.

2 Mathey DG, Decoodt PR, Allen HN, Swan HJC. The determinants of onset of mitral valve prolapse in the systolic click-late systolic murmur syndrome. Circulation 1976; 53: 872-8.

3 Roye JA, Basta LL. Echocardiographic pattern of mitral valve prolapse produced with special maneuvers in healthy individuals (abstract). Circulation 1975; 52, suppl II: 235.

4 Greene MA, Boltax AJ, Lustig GA, Rogow E. Circulatory dynamics during the cold pressor test. Am f Cardiol 1965; 16: 54-60.

5 Jeresaty RM. Mitral valve prolapse. New York: Raven, 1979: 53-60.

6 Devereux RB, Perloff JK, Reichek N, Josephson ME. Mitral valve prolapse. Circulation 1976; 54: 3-14.

Requests for reprints to Dr David Pitcher, Plymouth General Hospital, Plymouth, South Devon. 